\title{
Learning in the working place: the educational potential of a multihead microscope in pathology postgraduate training
}

\author{
Dominique Sandmeier • Fred Bosman • Maryse Fiche
}

Received: 14 July 2008 /Revised: 15 December 2008 / Accepted: 18 December 2008 / Published online: 14 January 2009

(C) Springer-Verlag 2009

\begin{abstract}
Training future pathologists is an important mission of many hospital anatomic pathology departments. Apprenticeship - a process in which learning and teaching tightly intertwine with daily work, is one of the main educational methods in use in postgraduate medical training. However, patient care, including pathological diagnosis, often comes first, diagnostic priorities prevailing over educational ones. Recognition of the unique educational opportunities is a prerequisite for enhancing the postgraduate learning experience. The aim of this paper is to draw attention of senior pathologists with a role as supervisor in postgraduate training on the potential educational value of a multihead microscope, a common setting in pathology departments. After reporting on an informal observation of senior and junior pathologists' meetings around the multihead microscope in our department, we review the literature on current theories of learning to provide support to the high potential educational value of these meetings for postgraduate training in pathology. We also draw from the literature on learner-centered teaching some recommendations to better support learning in this particular context. Finally, we propose clues for further studies and effective instruction during meetings around a multihead microscope.
\end{abstract}

D. Sandmeier $\cdot$ F. Bosman $\cdot$ M. Fiche

Institut Universitaire de Pathologie,

Centre Hospitalier Universitaire Vaudois (CHUV),

Université de Lausanne,

Lausanne, Switzerland

M. Fiche $(\square)$

Maryse Fiche Unité Pédagogique,

Faculté de Biologie et de Médecine,

Université de Lausanne,

Bugnon 211011 Lausanne, Switzerland

e-mail: maryse.fiche@chuv.ch
Keywords Pathology training · Postgraduate training · Microscope - Workplace learning - Apprenticeship . Socialization

\section{Introduction}

The quality of future pathologists training is paramount for quality of patient care. The competencies to be acquired include knowledge - a complex repertoire of images, concepts, and terminology, skills, and attitudes. Postgraduate training is part of the mission of hospital anatomic pathology departments. The educational approach in use in most programs includes, like for other medical specialties, "learning by doing," and specifically doing together with senior pathologists, an approach defined as companionship. As pointed out by Swanwick '(...) the bulk of learning is expected to take place at, through or from work' [1-3].

Though apprenticeship is highly valued as a method for various kinds of learning, the tight intrication of training and daily work in pathology departments may hamper an adequate recognition of the educational process embedded in diagnostic tasks [4]. Paying attention to quality of training in this context requires first to recognize educational opportunities and to understand how learning occurs and can be supported. A few reports have addressed this issue, so far, in the specific context of pathology postgraduate training $[5,6]$.

In our institution, the Lausanne University Pathology Institute, like in a multitude of similar institutes, a multihead microscope (MHM) is used for daily meetings to discuss and share problematic cases but also for other kinds of collaborative work involving also technicians, clinicians, and researchers [7]. This tool is used in complement of other practices, around individual or double-headed microscopes 
and use of digital images on computers or projection screens. While observing pathologists' meetings around the MHM, numerous and an old tradition in our institution, we realized that this setting in which a small group of professionals with various levels of expertise, interactively discuss authentic cases, presented several of the characteristics of effective learning as defined in current medical education conceptions. This was the starting point of the observations and reflections presented in this paper. Our aim is to draw the attention of postgraduate training supervisors on the educational potential of the multihead microscope, which can be optimized once its educational potential is recognized.

\section{Methods}

Two authors (DS, MF) first shared the observations they had gathered as participants in meetings around the MHM at the Lausanne University Pathology Department, during, respectively, 15 and 6 years of daily attendance. This informal observation was pursued prospectively during one more year by one author (MF), with a focus on trainers and trainees' roles and interactions. The findings were confirmed by a third observer (FB), also a frequent attender of these meetings for more than 15 years. We then reviewed the literature on effective learning and teaching in order to explain why, and under which conditions, and requirements, the meetings around the MHM provide a high educational value for postgraduate pathology training.

\section{The multihead microscope: a venue for learning and socialization}

The MHM, for multihead microscope, is a central tool at the University Institute of Pathology of the Lausanne University, in Switzerland. A lot of people come around it, looking at microscopic preparations through its 13 appendiceal eyes which make it look like an octopus
[Fig. 1]. All day long, different groups successively sit around the MHM, either for fixed appointments or for more spontaneous and informal ones [7]. From its permanent though silent and central position, the MHM witnesses the daily life of the Pathology Institute as a privileged observer of an important process going on in the institution: the perpetual renewal of newcomers and their progressive transformation into "old-timers" [4].

Four days a week, at 8:15 A.M., about 15 pathologists, senior, residents, and clerks, meet around the MHM for about 45 min - just like in a clinical morning round. Some junior or senior pathologists present current typical or challenging diagnostic cases from different subspecialty domains (gastrointestinal tract, pediatrics, soft tissues, and the like) to the whole medical staff. After a brief clinical history and a microscopic description, a differential diagnosis is exposed, and the clues for the diagnosis are debated. Most of the time, presented cases trigger questions, discussions, and different understandings are confronted. If present, a specialist of the domain will usually provide elements of evidence or new insights to clarify the debate, or someone will pick a textbook. Friday morning meetings are dedicated to reviewing systematically the frozen section diagnosis of the previous week, and two Thursday meetings per month to discussing ten challenging cases sent from one of the nine other institutes participating in the "Kansas" international seminar series initiated by Ivan Damjanov from Kansas City (Kansas, USA). Whatever the level of pathology knowledge, each participant can improve his diagnostic experience through these meetings. However, older voices are heard more often than younger ones. Some immediately venture a diagnosis, while others tend to seek opinions in asking questions. Many junior attendees are rather silent; though, they do come everyday and seem to appreciate these meetings.

The end of these morning meetings around the MHM offers a frequently used opportunity to make announcements and discuss domestic topics, organizational decisions, or various other issues. Resulting discussions

Fig. 1 Senior and junior pathologists working around the multihead microscope

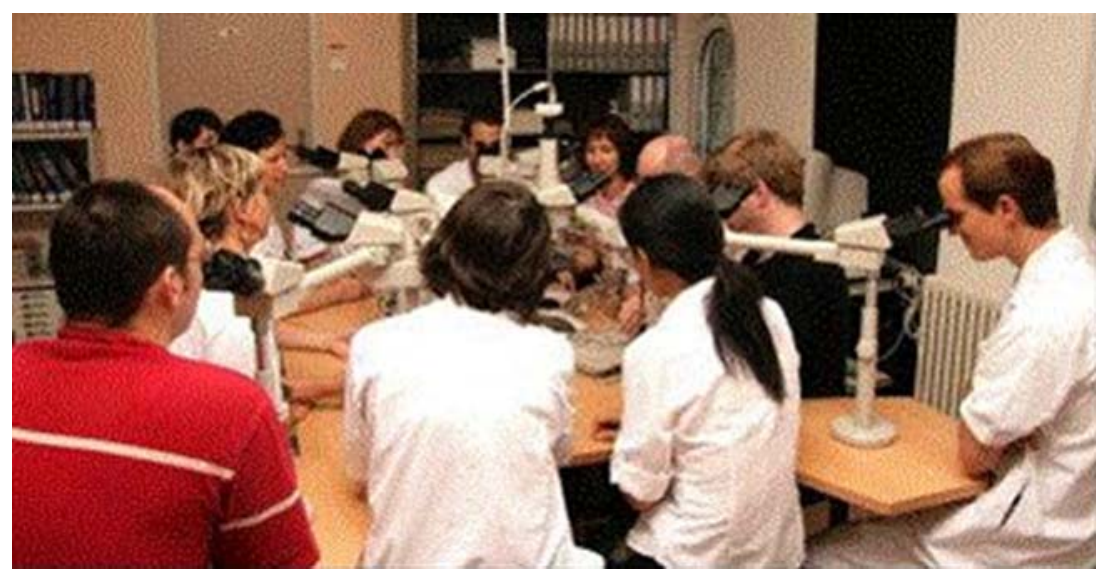


represent an opportunity for socialization of trainees in all aspects of the life of a histopathology department.

Other types of meetings are also held on a regular basis. Weekly, one pathologist and two cytologists meet for $1 \mathrm{~h}$ to confront cytology and histology results for the same patients, for the purposes of the internal quality control process, often with the participation of one or more residents. Some diagnoses are revised, and the basis for revision is clarified. A common culture of learning from mistakes is progressively built. Once a week too, a formal teaching session for pathology trainees takes place. Within a program elaborated on a yearly basis by trainees and supervisors together, senior pathologists prepare, in turn, a set of didactic sections on a given theme. The residents try to elaborate a diagnosis before the meeting, using their prior knowledge as well as textbooks, articles, or established guidelines. Then, around the MHM and with the aid of faculty and peers, the cases are reviewed, diagnostic hypotheses and relevant ancillary techniques are debated.

Once a month, a clinicopathologic correlation meeting in general surgery is held around the MHM. Clinicians select difficult cases, while the responsible pathologist presents the related histopathology. These meetings aim at bringing light to the interpretation of the whole clinical history and emphasize the role pathology plays in clinical management. By joining around the microscope, clinicians and residents progressively understand the pitfalls and limitations of morphology, whereas a detailed clinical history helps the pathologist interpreting difficult lesions. The dialogue between clinicians and pathologists is enhanced, and the reciprocal trust grows. During these meetings too, the MHM observes different types of participation: older attendees tend to more often ask questions, raise objections, while younger participants are more silent. A similar clinicopathological meeting around patients' biopsies sections is also held weekly for renal transplants biopsies.

By these meetings, pathologists are repeatedly exposed to a variety of microscopic images presented in their clinical context, and to peer discussion in small groups and in an informal atmosphere, characteristics which meet several of the conditions for effective learning.

\section{Learning around the MHM}

What do trainees learn around the MHM?

As presenters, they will get experience in exposing a case in a clear and understandable voice and a logical sequence. They will read prior to the session to collect additional information on the disease they present. As nonpresenters, they will increase their repertoire of images and associated vocabulary. The set of competencies they will build through repeated attendance also includes professional skills like framing a diagnostic problem, reaching a consensus through peer discussion, searching information, acknowledging new data, or changing concepts. They will progressively acquire a relevant idea of areas of diagnostic uncertainty and of pitfalls and hopefully an attitude toward best diagnostic accuracy.

How does learning occur?

The MHM presents, as an educational tool and context, several characteristics expected, according to sociocognitive theories of learning, to support its efficiency for professional training.

First, learning around the MHM fulfils the conditions for situated learning in the meaning attributed to this term by Lave and Wenger in their work entitled "Situated learning and peripheral participation" [4]. Around the MHM, participants learn from real cases, discussed by physicians with the responsibility to reach a diagnosis and sign a report. Within Lave and Wenger's theory, this staff around the MHM represents a "community of practice" sharing common professional constraints, rules, and language. Included in this concept is peripheral participation: as in traditional forms of apprenticeship, newcomers first participate by observing the process - the type of cases shown, presenters' behaviors, how discussions are led [4]. Later on, as their knowledge and level of responsibility in the diagnostic workload grows, they progressively move to a more central participation, as presenters and active discussants. Learning under such conditions is considered as highly efficient [4].

The way residents learn around the MHM is expected to be efficient also according to the perspective of cognitive psychology [8-11]. Within cognitive psychology, learners actively build their knowledge, in a cumulative process, on the basis of what they already know. Memory is crucial to the process and divided into short-term and long-term parts, with limited and unlimited capacity, respectively [8-11]. The way knowledge is acquired and organized for storage in long-term memory is of highest importance for future recall and application. From this perspective, learning around the MHM offers at least three favorable conditions for efficient learning. First, and close to the concept of situated learning, knowledge is acquired in a relevant context, like current cases representative of future relevant professional problems. Thus, it is stored in the learner's memory in association with other pieces of the context, which enhances the chances of adequate retention and further recall in similar contexts. Second, deliberation on knowledge, as it takes place during discussions around images and concepts, is considered to favor deep learning, at higher levels [10]. This happens when, through questions 
and comments, learners elucidate links between prior and new knowledge, and overcome partial or misunderstandings. Deliberation on knowledge contributes to the building of a robust basis of images, vocabulary, concepts, older and new insights, adequately structured around relevant problems, similar to the ones these trainees will face in their future practice. Finally, as demonstrated by sustained residents' attendance, trainees view meetings around the MHM as interesting moments that sustain their motivation, another essential element of efficient learning according to cognitive psychology [8-11].

Finally, and most importantly, the MHM is a venue for reflective practice and education of reflective physicians, according to the concept developed by Schön [12]. According to Schön, professional competence is a particular and complex type of knowledge which can be best developed through the process of reflection, including critical retrospective examination of one's practice, with its positive or negative outcomes, confrontation to some sort of reference framework, and peer discussion [12]. Around the MHM, trainees can observe how seniors model the skills and attitudes of reflection in and on action and thus learn an important dimension of professional practice. This process is especially at work during quality control sessions around the MHM, during which ancient cases are reviewed, sometimes in confrontation with further documentation such as additional specimen or clinical outcome.

Unique educational advantages of the multihead microscope

The multihead microscope allows trainees to learn from:

Broadened exposition to microscopic images with peer discussion

Accumulated experience in real-life daily cases

Active involvement in cases search and presentation

Observation of senior pathologists' practice in image analysis,

diagnostic reasoning, and consensus building

Development of a reflective attitude and an awareness of pitfalls, risks of errors, and difficult diagnostic areas

\section{Teachers around the MHM}

What do senior pathologists do around the MHM?

They attend, present cases, or encourage residents to do so; some of them enrich the institution's caseload with their external consultation cases; they bring their experience and medical knowledge to the discussion, reorient a debate, redress misunderstandings and false diagnosis; by the manner they intervene in the discussion, they hopefully contribute to create a challenging and nonthreatening learning climate.
Is that teaching?

Though few senior physicians do consider that they teach in this context (personal observations), it is quite clear that they support residents' learning around the MHM, even in meetings which are not part of a structured instructional program. MHM meetings are generally viewed primarily as part of the diagnostic work and reaching the right diagnosis is a main concern for the group. Demonstration of content expertise, including image recognition, is the contribution expected from the more experienced participants in this perspective. On the basis of what we have described above, however, MHM sessions do also have an important educational potential, in line with the educational mission of pathology departments in academic hospitals within the companionship framework [1-4]. The common accomplishment, by trainees and supervisors, of daily tasks related to patient care represents the major teaching responsibility of pathology faculty, be it ill defined and insufficiently recognized, much more important than formal teaching sessions in terms of amount of time spent. The growing attention paid to the quality of postgraduate training should include recognition of the educational importance of physicians as postgraduate educators and the need for explicit training for and evaluation and rewarding of this role [13-15]. Recognizing potential learning and teaching moments within daily activities of a Pathology Institute, and fostering attitude and behavior for their optimal use is one of the challenges of companionship [1-3]. However, acknowledging MHM sessions as no longer diagnostic-only but also educational moments, certainly does not imply turning these sessions into lectures!

\section{Improving teaching around the MHM}

The following suggestions, derived from authors' informal observations as well as from the literature on effective learning and teaching in medical education, would have to be supported by further studies. We expect, however, that they could serve as a starting guide for faculty willing to improve their participation as educators around a MHM.

In a student-centered perspective, teachers should care about what learners do $(16,17)$. Trainees learn more and better when they are exposed to numerous cases, selected for their level of training, be they common - but archetypical, or more exceptional; when they actively engage in the diagnostic and bibliographic research, for a case in which they have a responsibility; when they take the opportunity to present a case and receive constructive feedback adapted to their level, in a nonthreatening climate and respectful manner [13, 18]. Trainees benefit from senior pathologists appropriately demonstrating well-structured, clinically rele- 
vant and up-to-date pathology knowledge, as well as modeling rigorous and methodical reasoning, enthusiasm, curiosity, and constructive peer-discussion skills [13, 19]. Their knowledge basis is reinforced by interventions addressing the structure of knowledge, rather than the accumulation of isolated detailed facts, as well as those recalling important basic diagnostic processes, which they can re-use in their future practice. They benefit from seniors triggering a debate ("why?"), broadening the discussion, or closing it by a short synthesis in due time [13-16]. Teachers should also be aware of who they are and why they sit around the MHM.

Trainees get frustrated when discussions start from implicit diagnostic hypotheses, understood only by a few senior physicians, when the right diagnosis is given away without time for discussion. They do not necessarily learn from seniors exposing very specialized knowledge nor do they value inter-senior fights.

\begin{tabular}{ll} 
Teacher around the MHM & \\
Dos & Don'ts \\
Provide cases & Neglect to come \\
Think of learners first & Forget novices in the audience \\
Select didactic cases & Go straight to the diagnosis \\
Respect the discovery process & Jump into inter-experts \\
in novices & discussion \\
Model explicit and systematic & Assume everyone already \\
presentation & knows \\
Encourage (and allow time for) & Forget the big picture \\
reasoning & \\
Favor structure, general rules & Overvalue details against \\
& the big picture \\
Ask or trigger relevant questions & Injure other participants \\
Reformulate and summarize & Despise not knowing (just \\
difficult points & not learning) \\
Care for the climate & \\
Be happy of everyone's learning & \\
\hline
\end{tabular}

\section{Perspectives for further work}

This paper presents an observation of the use of a MHM in a hospital pathology department, and a literature review supporting the analysis of its potential role as an educational format, with some resulting requirements for effective teaching in this context. In summary, the MHM is a venue for situated learning and reflective practice. Meetings around the MHM may represent fruitful learning and teaching moments within the context of apprenticeship, the dominant educational strategy for postgraduate pathology training. Clarifying the theoretical background of this approach is important to better understand, behind a traditional practice in the many institutions equipped with a MHM, an educational activity with specific requirements for effectiveness. Though knowledgeable senior pathologists are essential around the MHM, knowledge and seniority do not guarantee optimal educational approaches. These should be explicitly favored in managing MHM sessions. Further studies should address trainee perception of the actual contribution of meetings around the MHM to their training and explore how supervisors' effectiveness could be improved in this context.

Conflict of interest statement We declare that we have no conflict of interest.

\section{References}

1. Swanwick T (2005) Informal learning in postgraduate medical education: from cognitivism to 'culturism'. Med Educ 39: 859-865

2. Bleakley A (2002) Pre-registration house officers and ward based learning: a 'new apprenticeship' model. Med ed 36:9-15

3. Teunissen PW, Scheele F, Scherpbier AJ et al (2007) How residents learn: qualitative evidence for the pivotal role of clinical activities. Med Educ 41:763-770

4. Lave J, Wenger E (1991) Situated learning: Legitimate peripheral participation. Cambridge University Press, Cambridge, UK

5. West K (2005) Early training in Histopathology. Curr Diag Pathol $11: 317-322$

6. Syred KS (2005) Learning histopathology: a trainee's viewpoint. Current Diagnostic Pathology 11:323-328

7. Sandmeier D, Fiche M (2008) The educational benefits of a multihead microscope in an academic hospital. Med Teach 30:437-439

8. Shulman LS (1970) Cognitive learning and the educational process. J Med Educ 45:90-100

9. Shuell TJ (1986) Cognitive conceptions of learning. Rev Edu Res $56: 411-436$

10. Biggs JB (1991) Teaching for learning. The view from cognitive psychology. ACER, Victoria (Australia)

11. Regehr G, Norman GR (1996) Issues in cognitive psychology: implications for professional education. Acad Med 71:988-1001

12. Schön DA (1987) Educating the reflective practitioner: Toward a new design for teaching and learning in the professions. Jossey Bass, USA, San Francisco

13. Pratt DD, Arseneau R, Collins JB (2001) Reconsidering "good teaching" across the continuum of medical education. J Contin Educ Health Prof 21:70-81

14. Gordon J, Hazlett C, Cate OT et al (2000) Strategic planning in medical education: enhancing the learning environment for students in clinical settings. Med Educ 34:841-850

15. Kilminster S, Cottrell D, Grant J et al (2007) AMEE Guide $\mathrm{N}^{\circ} 27$ : effective educational and clinical supervision. Med Teach 29:2-19

16. Mann KV (2001) Thinking about learning: implications for principle-based professional education. J Contin Educ Health Prof 21:90-96

17. Weimer M (2002) Learner-centered teaching. Five key changes to practice. Jossey-Bass San Francisco

18. Billet S (2002) Toward a workplace pedagogy: guidance, participation and engagement. Adult Educ Q 53:27-43

19. Kenny NP, Mann KV, MacLeod H (2003) Role modelling in physicians' professional formation: reconsidering an essential but untapped educational strategy. Acad Med 78:1203-1210 